\title{
OA05-05. Impact of in vivo CD4 binding during HIV-I Env trimer immunizations of rhesus macaques
}

\author{
I Douagi*1, MM Forsell ${ }^{1}$, C Sundling ${ }^{1}$, S O'dell2 ${ }^{2}$ R Seder ${ }^{2}$, JR Mascola², \\ K Loré ${ }^{3}$, RT Wyatt ${ }^{2}$ and GB Karlsson Hedestam ${ }^{1}$
}

\author{
Address: ${ }^{1}$ Department of Microbiology, Tumor and Cell Biology, Karolinska Institutet, Stockholm, Sweden, ${ }^{2}$ Vaccine Research Center, National \\ Institutes of Health, Bethesda, MD, USA and ${ }^{3}$ Center for Infectious Medicine, Karolinska Institutet, Stockholm, Sweden \\ * Corresponding author
}

from AIDS Vaccine 2009

Paris, France. 19-22 October 2009

Published: 22 October 2009

Retrovirology 2009, 6(Suppl 3):O35 doi:10.1186/1742-4690-6-S3-O35

This abstract is available from: http://www.retrovirology.com/content/6/S3/O35

(C) 2009 Douagi et al; licensee BioMed Central Ltd.

\section{Background}

We recently reported that immunization with cleavagedefective soluble HIV-1 envelope glycoproteins (Env) trimers in monkeys, but not in rabbits, results in the elicitation of antibodies directed against the co-receptor binding site of gp120. This finding demonstrates that the high affinity interaction between Env and primate CD4 results in an alteration of Env immunogenicity. To further investigate the impact of Env-CD4 in vivo interactions during vaccination, we immunized rhesus macaques with wild type (wt) and CD4 binding defective Env trimers.

\section{Methods}

Groups of five adult rhesus macaques were immunized with wt trimers or CD4 binding defective trimers, with either retained or abrogated capacity to form the co-receptor binding-site. Plasma and PBMCs were collected 2 weeks post-immunization. Frequency of Env-specific B and $\mathrm{T}$ cells were assessed by ELISpot analysis and flow cytometry. Serum antibodies were measured by ELISA and neutralization assays. Four weeks following the last immunization, control and vaccinated animals were challenged with SHIVSF162P4.

\section{Results}

Antibodies against the co-receptor binding site were elicited in animals immunized with wt trimers, but not in animals immunized with the CD4-binding defective mutants. Elimination of the Env-CD4 in vivo interaction did not affect vaccine-induced Env-specific $\mathrm{T}$ cell responses or levels of total elicited binding antibodies. However, differences in the quality of the in vitro neutralizing antibody response were observed between the three groups. A comparable decrease in plasma viral loads compared to unvaccinated controls was also measured following challenge in all three groups.

\section{Conclusion}

These results confirm that the wt Env trimers used here interact with host CD4 in vivo affecting the quality of the elicited antibody response. As Env-based vaccines progress from small animals to primates, the effects of high affinity CD4 interaction may need to be considered in regards to immunogen design. 